\title{
Mechanical characterisation of a viscous-elastic plastic material, sensitive to hydrostatic pressure and temperature
}

\author{
V. D. Le ${ }^{1}$, M. Caliez ${ }^{1}$, M. Gratton ${ }^{1}$, A. Frachon ${ }^{1} \&$ D. Picart ${ }^{2}$ \\ ${ }^{1}$ Laboratoire de Mécanique et Rhéologie, EA 2640, \\ Ecole d'Ingénieurs du Val de Loire, \\ Université François Rabelais de Tours, Blois cedex, France \\ ${ }^{2}$ C.E.A. Le Ripault, Monts, France
}

\begin{abstract}
This paper deals with the characterization of the static mechanical behaviour of an energetic material. Due to the constituents (crystals and a polymeric binder), the behaviour is influenced by the pressure, the temperature and the strain rate. The temperature, considered varying slowly, is a parameter and the computational problems are uncoupled. Therefore, a complete experimental protocol and a model have been developed. Inspired from the Visco-Scram model, the behaviour is described using a general Maxwell model in which all the branches are affected by an isotropic damage. The first branch takes into account elastic-plastic behaviour. The yield stress is given by a parabolic criterion, characterized using compressive, tensile and tri-axial tests. The hardening is isotropic and the plastic flow rule is nonassociated. The other branches are viscoelastic. A genetic algorithm is used to optimise the viscoelastic parameters, previously obtained using DMA measurements. Comparisons between the model and experiments are proposed for different temperatures, strain rates and pressures. At last, a user material subroutine has been developed in Abaqus Standard and finite element computations of the Brazilian test are compared to the experimental response.

Keywords: energetic material, parabolic plastic criterion, genetic algorithm, DMA, viscoelasticity, Isotropic damage, Brazilian tests.
\end{abstract}




\section{Introduction}

The material is made of organic and energetic crystals mixed with a few percentage of a polymeric binder. After an isostatic compaction forming process, the material has a small porosity of a few percent. Samples can be machined in several geometric shapes, which are more than ten times the length of the material heterogeneity.

In order to survey the possible aging of this material, an accurate determination of the mechanical properties has to be done. Unfortunately, this material being available in small amounts, the characterization must be made using a reduced number of standard tests. An unusual experimental procedure is proposed in this paper for this kind of material. When monotonic loading paths were used to determine for example the influence of the strain rate, each sample is submitted to complex loading paths including relaxation, recovery and cyclic conditions.

The observation of loading-unloading diagram on figure 1 shows some of the main features of the material at room temperature, and entails specific arrangements for the mechanical tests. 1) Hydrostatic pressure sensitivity: to consider it, an initial hydrostatic loading path $(0 \mathrm{MPa}, 5 \mathrm{MPa}$ and $10 \mathrm{MPa})$ is made before the run of a uniaxial compression load. 2) Viscosity: different strain rates $\left(5.10^{-6} \mathrm{~s}^{-1}\right.$ to $\left.10^{-3} \mathrm{~s}^{-1}\right)$ have been used to observe such effect. The parameters of the viscoelastic part of the behaviour have been determined using a DMA apparatus (Dynamic Mechanical Analysis). 3) The plastic strains are determined using relaxation and recovery delays. 4) Initial elastic behaviour: standard tests made in various material directions show an initial isotropic behaviour. 5) Damaging: systematic cyclic loading-unloading programs have been performed. 6) Dispersion: to ensure a minimum statistical validity, each loading program is repeated five times.

The temperature is considered as a parameter in the model. The material been temperature dependent, compressive and tensile tests are perform at four different temperatures: $5^{\circ} \mathrm{C}, 20^{\circ} \mathrm{C}, 35^{\circ} \mathrm{C}$ and $50^{\circ} \mathrm{C}$. The figure 2 shows the effect of the temperature on monotonic compressive experiments.

The main models available in the literature ([1-3]) for this kind of material have been developed for transient dynamic behaviour and are not adapted for a quasi-static study. For example, the influence of the pressure is omitted, even as the difference of behaviour observed in tension and compression loading paths.

The Maxwell model we have chosen (fig. 3) is close to the constitutive law proposed in [2]. Several damageable viscoelastic branches and one damageable elastoplastic branch are used. The main difference with the Bennett and coworkers model is the presence of the elastoplastic branch.

The determination of the plastic (resp. viscoelastic) behaviour is described in the second (resp. third) part of this paper. In particular, a genetic algorithm has been used to optimize the determination of the viscoelastic parameters. The fourth part deals with the damage rule. It can be noted that the characterization of the plasticity, the damage and the viscosity are uncoupled. The damage rule is assumed to affect also the viscoelastic branches. 
Lastly, some comparisons are presented between the model response and the available experimental data.

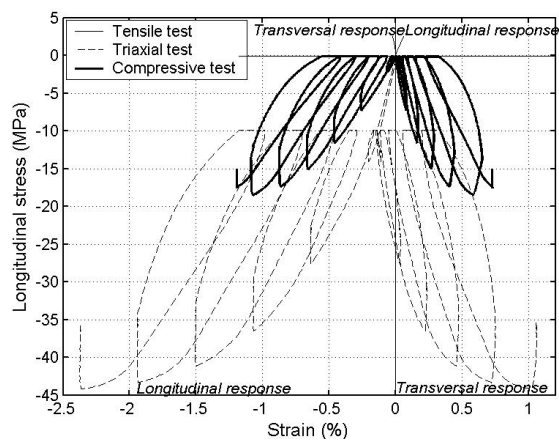

Figure 1: Cyclic tests for a given Figure 2: Compressive tests for a strain rate: pressure effect on the global responses of the material $\left(20^{\circ} \mathrm{C}\right)$.

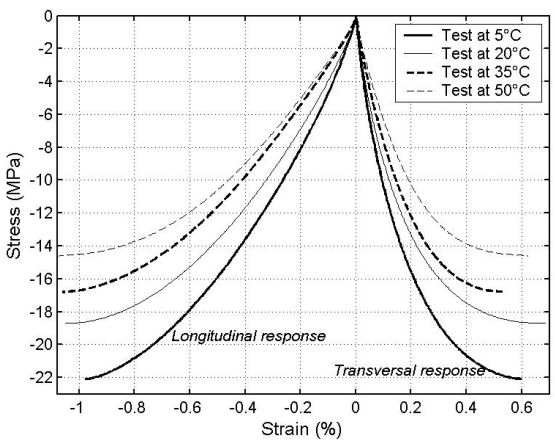
given strain rate: temperature effects.

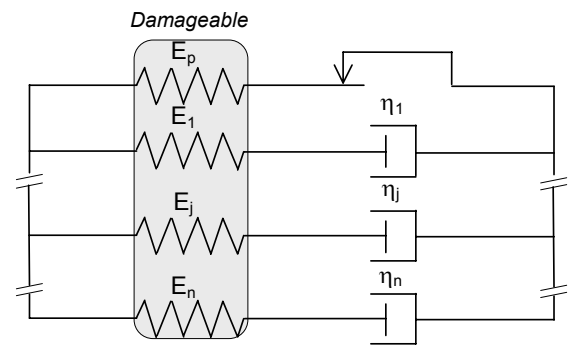

Figure 3: Rheologic diagram of the viscoelastic plastic damageable model.

The test procedure is described in [4]. The first stage (when this one exist) of the loading program is a hydrostatic loading phase. Then, five or six uniaxial loading-relaxation-unloading-recovery cycles are done. The test is driven by one of the two longitudinal gages. Relaxation times have been defined to guarantee an almost complete relaxation of viscous stresses. All the tensile tests and the compressive tests have been done for several temperatures $\left(5^{\circ} \mathrm{C}, 20^{\circ} \mathrm{C}, 35^{\circ} \mathrm{C}\right.$ and $\left.50^{\circ} \mathrm{C}\right)$.

Some compressive DMA experiments have been realized using samples of $50 \mathrm{~mm}$ long, and a cross section of $4 \times 4 \mathrm{~mm}^{2}$. A small initial preload of $10 \mu \mathrm{m}$ and a strain amplitude of $5 \mu \mathrm{m}$ are used to stay in the viscoelastic domain. The range of frequencies going from 0.004 to $40 \mathrm{~Hz}$, the strain rate ranges from $2.10^{-6} \mathrm{~s}^{-1}$ to $2.10^{-2} \mathrm{~s}^{-1}$. 


\section{Elastoplasticity}

The macroscopic stress tensor $\underline{\underline{\sigma}}$ is defined as the sum of the stress of each branch:

$$
\underline{\underline{\sigma}}=\underline{\underline{\sigma}}_{e p}+\sum_{j=1}^{n} \underline{\underline{\sigma}}_{j},
$$

where the subscript "ep" (resp. " $j$ ") denoted the elastoplastic branch (resp. the $\mathrm{j}^{\text {th }}$ viscoelastic branch). An additive decomposition is assumed for the strain mechanical part (superscript "m") and the strain thermal part (superscript "th"):

$$
\underline{\underline{\varepsilon}}=\underline{\underline{\varepsilon}}^{m}+\underline{\underline{\varepsilon}}^{t h} \text {. }
$$

An additive decomposition of the mechanical strain of each branch is also assumed between: an elastic part (superscript "e") and a plastic (superscript "p") part (resp. viscous) (superscript " $p$ " or “v”):

$$
\begin{aligned}
& \underline{\underline{\varepsilon}}^{m}=\underline{\underline{\varepsilon}}_{e p}^{e}+\underline{\underline{\varepsilon}}_{e p}^{p} \text { elasto-plastic branch, } \\
& \underline{\underline{\varepsilon}}^{m}=\underline{\underline{\varepsilon}}_{j}^{e}+\underline{\underline{\varepsilon}}_{j}^{v} \text { visco-elastic branches. }
\end{aligned}
$$

\subsection{Thermo-elasticity}

The elastic part is supposed linear damageable and given by the following equation:

$$
\underline{\underline{\sigma}}_{e p}=(1-d) \mathrm{E}(\theta): \underline{\underline{\varepsilon}}_{e p}^{e},
$$

where $\mathbf{E}(\theta)$ is the elastic tensor of the virgin material and is defined as a function of the temperature, and $d$ a damage parameter (see below). To identify the elastic mechanism, we need to isolate the elastoplastic behaviour (in particular to be sure that the viscous stresses are relaxed). To this end, the ends of the relaxation and recovery phases are used to determine the elastic modulus. The initial Young's modulus $E_{e p}$ is a function of the temperature and is defined in the table 1.

A value of 0,3 is obtained for the Poisson's ratio for all the temperatures, using longitudinal and transversal strain measurements. This ratio is used for all the viscoelastic branches. The thermal expansion coefficient is $c=50.10^{-6} \mathrm{~K}^{-1}$.

Table 1: $\quad$ Temperature dependency of the Young modulus.

\begin{tabular}{|c|c|c|c|c|}
\hline Temperatures & $5^{\circ} \mathrm{C}$ & $20^{\circ} \mathrm{C}$ & $35^{\circ} \mathrm{C}$ & $50^{\circ} \mathrm{C}$ \\
\hline Initial Young modulus $E_{e p}(\mathrm{MPa})$ & 3400 & 2900 & 2700 & 2450 \\
\hline
\end{tabular}

\subsection{Yield criterion}

A review of the main criteria used to describe isotropic plasticity is presented in [4]. A criterion has been developed at Cambridge University in view of soil modeling and is famous today as the "Cam-clay" model [5]. Numerous 
adaptations of this model were then developed for various applications [6-8]. As the forming process of the material is an isostatic compaction up to a pressure of $200 \mathrm{MPa}$ (which amplitude will never be reached in quasi-static applications), the criteria is supposed open on the hydrostatic negative axis. Open threshold are usually derived from Mohr-Coulomb, Mises-Schleicher [9], Drucker-Prager [10] and more recently Hoek-Brown formulations [11]. Raghava et al. [12] applied the Mises-Schlecher's threshold to polymers. The evolution of this criterion is described by two hardening variables, associated to tensile and compressive response. Lastly, a unified model is proposed by Aubertin and Li [5] in order to reproduce all the kinds of criterion (elliptic, parabolic, hyperbolic).

Due to a lack of data about the nature of the hardening mechanisms, an isotropic hardening parameter, denoted $k$, is introduced in the model. Then, a saturation of the hardening mechanism at the maximum stress is taken into account in the model. A nonlinear plasticity criterion reproduces the evolution of the yield stress (fig. 4). The following relation is used:

$$
f(Q, P, k)=\sigma_{e q}-k=0 \quad \text { with } \quad \sigma_{e q}=\sqrt{Q^{2}+\frac{k^{2}}{X(k)} P} \text {, }
$$

where $Q=\sqrt{(1 / 3) \underline{\underline{\sigma}}^{D}: \underline{\underline{\sigma}}^{D}}$ is the octahedric stress, $\underline{\underline{\sigma}}^{D}$ the deviatoric stress and $P$ the pressure. The set of yield curves is completely defined as soon as the function $X(k)$ and the hardening law are defined.

The following guidelines help for the determination of the function $X(k)$. First, it is assumed that the yield curves do not cross themselves in the $P-Q$ plane, each one being embedded in those of higher levels, all of them being embedded in the extreme curve. This is a necessary - but not sufficient condition for the phenomenon to be governed by a unique state variable which is the isotropic strain-hardening parameter. Elementary algebra shows that the following relation satisfies the previous assumption:

$$
X(k)=X_{0}+\left(X_{m}-X_{0}\right) \frac{k-k_{0}}{k_{m}-k_{0}},
$$

where the parameter $X_{0}=1,5 \mathrm{MPa}$, positioning the summits of the initial parabola, is supposed not being temperature depending, and the three parameters $X_{\mathrm{m}}, k_{0}$ and $k_{\mathrm{m}}$ are temperature depending. The $X_{\mathrm{m}}$ and $k_{\mathrm{m}}$ parameters are determined using the ultimate yield stress curve relating the maximum stress states in the $P-Q$ plane. The values are given table 2 .

Table 2: $\quad$ Temperature dependency of the hardening parameters.

\begin{tabular}{|c|c|c|c|c|}
\hline Temperatures & $5^{\circ} \mathrm{C}$ & $20^{\circ} \mathrm{C}$ & $35^{\circ} \mathrm{C}$ & $50^{\circ} \mathrm{C}$ \\
\hline$X_{\mathrm{m}}(\mathrm{MPa})$ & 1,635 & 1,620 & 1,610 & 1,592 \\
\hline$k_{0}(\mathrm{MPa})$ & 0,80 & 0,55 & 0,45 & 0,35 \\
\hline$k_{m}(\mathrm{MPa})$ & 3,88 & 3,5 & 3,26 & 2,82 \\
\hline$c_{1}$ & 700 & 500 & 500 & 500 \\
\hline$c_{2}$ & 150000 & 100000 & 100000 & 90000 \\
\hline
\end{tabular}


The hardening parameter $k$ has to be related to an effective plastic strain variable, denoted $p$. In order to obtain a unique curve $k(p)$ for all the available experimental data, $p$ is defined as the cumulated deviatoric plastic strain (fig. 5).

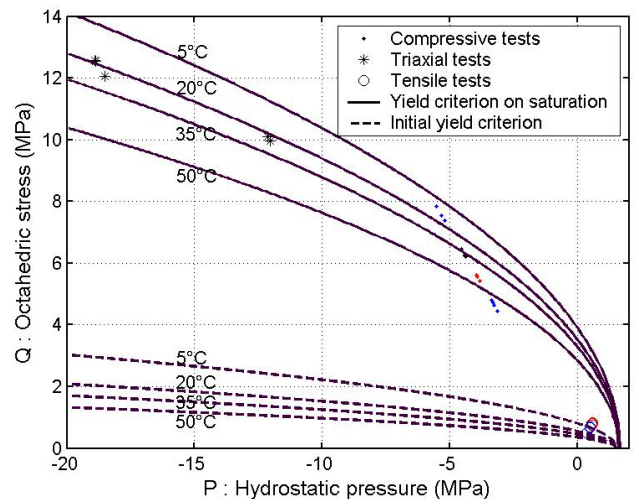

Figure 4: Initial and saturated yield criterions for the different temperatures.

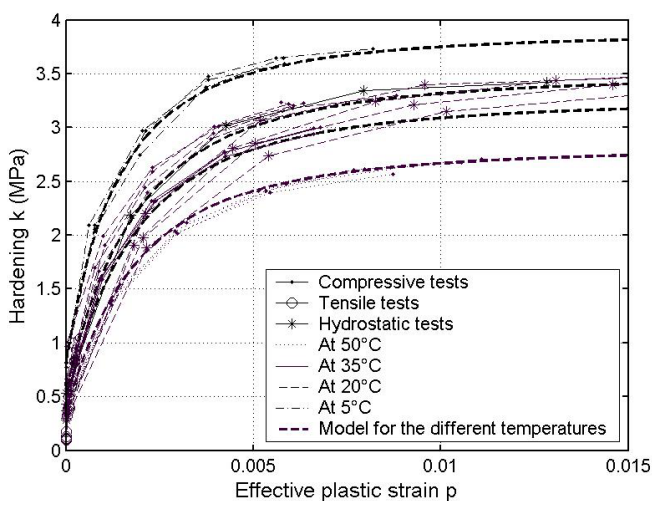

Figure 5: Hardening parameter $\mathrm{k}$ versus the effective plastic strain $\mathrm{p}$ for the different temperatures.

For the hardening law, the following hyperbolic relation is used to interpolate the data:

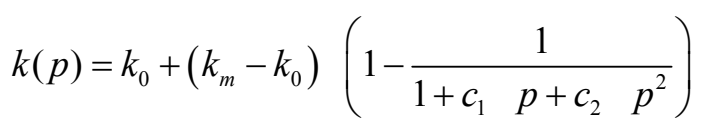

where $c_{1}$ and $c_{2}$ are two parameters temperature depending (table 2). 


\subsection{Flow rule}

The flow direction is determined using the ratio between volumic and deviatoric effective plastic strain rates. This ratio, usually called "dilatancy" and denoted $\beta$, is given by the following relation:

$$
\beta=\frac{\dot{\varepsilon}_{e p}^{p V}}{\dot{\varepsilon}_{e p}^{p D}}, \quad \text { with } \dot{\varepsilon}_{e p}^{p V}=\operatorname{Tr}\left(\underline{\underline{\dot{\varepsilon}}}_{e p}^{p}\right) \quad \text { and } \quad \dot{\dot{\varepsilon}}_{e p}^{p D}=\sqrt{3 \underline{\underline{\dot{\varepsilon}}}_{e p}^{p D}: \underline{\dot{\varepsilon}}_{e p}^{p D}}
$$

$\underline{\underline{\varepsilon}}_{e p}^{p D}$ being the deviatoric plastic strain rates.

The flow rule expression is then:

$$
\stackrel{\dot{\varepsilon}}{=}_{e p}^{p}=\dot{\lambda}\left(\frac{\underline{\underline{\sigma}}_{e p}^{D}}{3 Q}+\frac{\beta}{3} \underline{I}\right) \cdot \sqrt{\frac{3}{\left(1+\beta^{2}\right)}},
$$

$\dot{\lambda}$ being the plastic multiplier and the plastic flow direction being normalized. The dilatancy $\beta$ is characterized from the experimental results as a constant $(\beta=0,15)$. As a result, a nonassociated plastic law is justified.

\section{Viscoelasticity}

\subsection{Dynamic Mechanical Analysis}

DMA experiments are used to make a first identification of the linear viscoelastic parameters. The stress response to a unit sinusoidal strain solicitation for this kind of model can be break up in an in-phase part (related to the storage modulus $E_{\text {stor }}$ ) and an out of phase part (related to the loss modulus $E_{\text {loss }}$ ). The analytical response of a generalized Maxwell model is known and depends of the distributions of the Young modulus $\left(E_{j}\right)$ values and of the viscosity parameters $\left(\eta_{j}\right)$ values. In order to limit the number of parameters (here $n=10$ branches) for a more accurate determination, the following relations are proposed from the DMA results [4]:

$$
\eta_{j}=10^{\left(A_{1} \frac{j-1}{n-1}+A_{2}\right)} \text { and } E_{j}=A_{3}+A_{4}\left(e^{\left(A_{5} \frac{j-1}{n-1}\right)}-1\right) .
$$

Then, the number of unknowns decreases to five $\left(A_{1}\right.$ to $\left.A_{5}\right)$. These relations allow reproducing at the same time the storage and the loss modulus.

\subsection{Genetic algorithm}

The previous set of parameters is used here to determine the bounds of each parameter. We are in the case of a combinatory optimisation problem where a large number of solutions could be suitable. We have chosen to perform an inverse identification of the viscoelasticity parameters directly from the experimental tests. Classical optimisation methods, like conjugated gradient, 
have been dismissed because of the possible large number of solutions. Then, a genetic algorithm [13] has been used in this study.

Genetic algorithms are based on the Darwinian principle of "survival to the fittest". An initial population of a given size is created from a random selection of parameters values. Each parameter set represents individual chromosomes. Each individual is assigned of a fitness based on how well each individual chromosome allows it to perform in its environment. The algorithm produces new generations by applying three evolution operators: selection, crossover and mutation. For each generation, the fit individuals survive and the weak die. Evolution operators create new individuals (children) from two selected parents, and these children replace the weak individuals for the next generation. Successive generations are created until very fit individuals are obtained. This algorithm offers the advantage of exploring all the solutions space to find a global optimum of an optimization problem. A sensitivity analysis of the parameters is not required.

\subsection{Objective function}

The objective function is a direct measure of the quality of a solution. The goal is here to minimize the gap between the experimental strain-stress curves and the corresponding simulated curves. Due to different times of recording during the experiments, we propose a fitness function that represents the spatial gap, weighted by the segment length $L_{\mathrm{i}}$ between two consecutive data, in the stressstrain space, i.e.:

$$
O b j=\sum_{i=1}^{n b \exp p t s} \frac{\sqrt{\left(\sigma_{i}^{e}(t)-\sigma_{i}^{s}(t)\right)^{2}} \cdot L_{i}}{\sum_{i=1}^{n b \exp p t s} L_{i}},
$$

where superscripts "s" and "e" respectively denote simulations and experiments.

\subsection{Results}

The simulation uses the data corresponding at the end of load, the end of relaxation, the end of discharge and the end of recovery. From the values of the longitudinal strain and time in these points, the strain rate is rebuilt, constant by piece. The strain increment is then given from the strain rate and the step of time of the program.

At $20^{\circ} \mathrm{C}$, all the tests (compressive tests with different strain rates, tensile tests and triaxial tests) are used to optimise the five parameters $A_{\mathrm{i}} .60$ generations constituted of 500 individuals have been performed and leads to the optimized values of the five $A_{\text {i }}$ parameters at $20^{\circ} \mathrm{C}$. The restricted quantity of material does not allow making all those tests for all the tested temperatures. So, for the 5, 35 and $50^{\circ} \mathrm{C}$ temperature values, only the tensile and compressive tests, for only one strain rate, are done. Therefore, we supposed that the temperature dependency of the Young modulus of the viscoplastic branches is the same that the elastoplastic branch one: $E_{j}(\theta)=E_{e p}(\theta) \cdot E_{j}\left(20^{\circ} \mathrm{C}\right) / E_{e p}\left(20^{\circ} \mathrm{C}\right)$. 
Therefore, for the tests at $5^{\circ} \mathrm{C}, 35^{\circ} \mathrm{C}$ and $50^{\circ} \mathrm{C}$, only the parameters $A_{l}$ and $A_{2}$ have to be identified with the genetic algorithm. The results are presented in the table 3 .

Table 3: Temperature dependency of $A_{\mathrm{i}}$ parameters defining the $\eta_{j}$ viscosity parameters.

\begin{tabular}{|c|c|c|c|c|}
\hline Temperature & $5^{\circ} \mathrm{C}$ & $20^{\circ} \mathrm{C}$ & $35^{\circ} \mathrm{C}$ & $50^{\circ} \mathrm{C}$ \\
\hline $\begin{array}{l}\text { Tests com- } \\
\text { puted from: }\end{array}$ & $\begin{array}{l}\text { Compressive, } \\
\text { one strain rate }\end{array}$ & All & $\begin{array}{c}\text { Tensile/Compressive, } \\
\text { one strain rate }\end{array}$ & $\begin{array}{c}\text { Tensile/Compressive, } \\
\text { one strain rate }\end{array}$ \\
\hline$A_{1}$ & 2,78 & 3,98 & 2,15 & 2,62 \\
\hline$A_{2}$ & 2,63 & 1,64 & 2,60 & 2,32 \\
\hline$A_{3}$ & & 160,31 & & \\
\hline$A_{4}$ & & 16,15 & & \\
\hline$A_{5}$ & & 1,21 & & \\
\hline
\end{tabular}

\section{Isotropic damage}

Assuming an isotropic damage, experimental data shows that the phenomenon regularly increases with the highest positive principal strain (fig. 6). This observation indicates that the most probable damaging mechanism is the result of the development of internal micro-defects (cavities, cracks) with tension [1418]. A damaging factor $d$ is classically defined as:

$$
d=\frac{E_{0}-E}{E_{0}},
$$

where $E_{\mathrm{o}}$ and $E$ are the initial and current Young's modulus. A constant Poisson's ratio is assumed here.

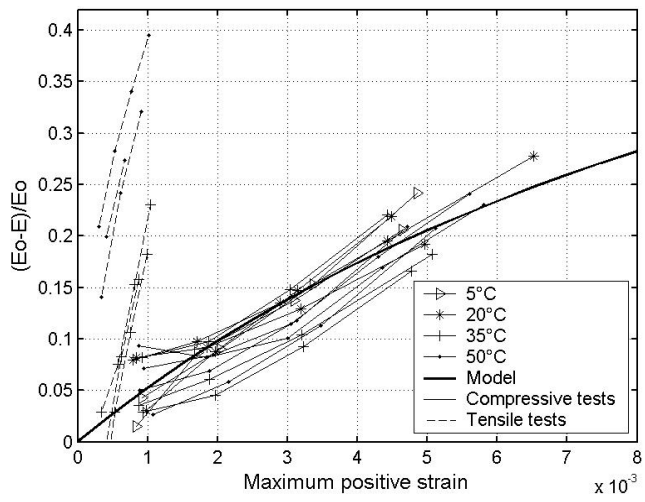

Figure 6: Damage versus the maximum positive strain.

Experimental values of $d$ immediately result from the measurements of $E$. An hyperbolic relation, eqn. (14), is used to reproduce an average evolution of 
the damaging factor, providing that its value is bounded to $1 ; d_{1}, d_{2}$ and $d_{3}$ are three parameters. The subscript "+" means "the positive part".

$$
d=(d_{1} \cdot \underbrace{\sup }_{\text {time }}<\underbrace{\max }_{I=1 \text { to } 3}\left|\varepsilon_{\mathrm{I}}\right|>_{+}+d_{2}) \cdot\left(1-\frac{1}{1+d_{3} \underbrace{\sup }_{\text {time }}<\underbrace{\max }_{I=1 \text { to } 3} \varepsilon_{\mathrm{I}}>_{+}}\right),
$$

The damage rule is reported in the fig. 6. One can see that the model response is identified using the compression measurements. The hydrostatic data are not taken into account because the pressure stops an eventually growth of the microcavities. The introduction of a second damage mechanism, as proposed in [19], to model the tensile damage is in study. The identified values are the same for all the temperatures: $d_{1}=0,5, d_{2}=0,75$ and $d_{3}=75$.

\section{Model versus experiments}

The constitutive law has been implemented in the finite element code Abaqus/standard. The model is compared to experimental data with unloading cycles to access to the plasticity level and the damage level. Those seem quite well reproduced even if the transversal model response does not present enough damage in compression (fig. 7-8). This observation can be associated to an anisotropic damage (which has been neglected here).

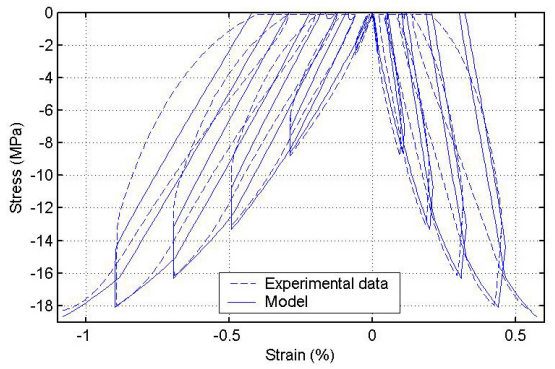

Figure 7: Compressive test $\left(3.10^{-6} \mathrm{~s}^{-1}\right.$; $\left.20^{\circ} \mathrm{C}\right) ; \quad$ model versus experiment.

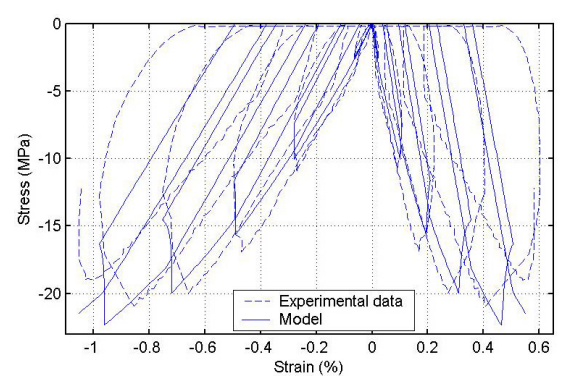

Figure 8: Compressive test (1.5 10$\left.{ }^{3} \mathrm{~s}^{-1} ; 20^{\circ} \mathrm{C}\right)$; model versus experiment.

The plasticity branch reproduces very well the pressure effect that causes the difference between tensile and compressive responses (fig. 7, 9, 10). The rate effect is also quite well reproduced on the two compressive tests even if the unloading curves do not present the same nonlinearity (fig. 7-8). In the same manner, the viscous effects in the triaxial test with $10 \mathrm{MPa}$ of confinement pressure (fig. 10) is underestimated. Certainly, these phenomenons is associated to an internal friction in the material or a viscosity pressure dependency. The pressure dependency is certainly the reason of the discontinuity of the $A_{i}$ 
parameters at $20^{\circ} \mathrm{C}$ (table 3 ), temperature for which all the tests are computed and in particular the triaxial ones.
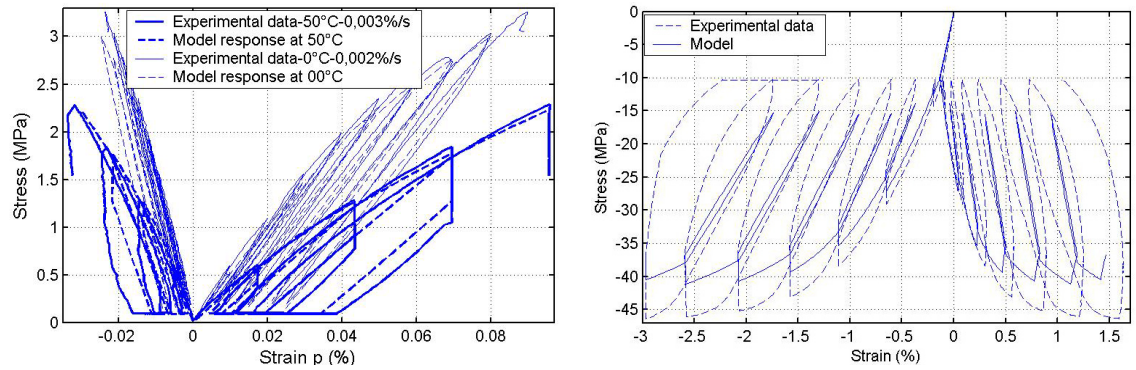

Figure 9: Tensile test $\left(3.10^{-5} \mathrm{~s}^{-1}\right)$; model versus experiment.

Figure 10: Triaxial test $\left(3.10^{-5} \mathrm{~s}^{-1}\right.$; $\left.20^{\circ} \mathrm{C}\right)$; model versus experiment.

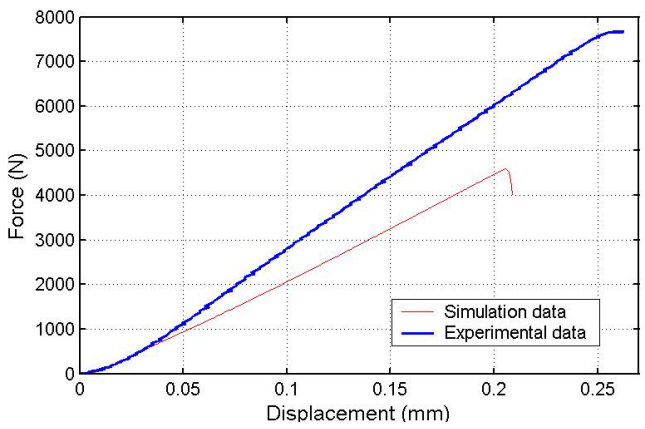

Figure 11: Global response of the Brazilian test; model versus experiment.

The temperature dependency of the model parameters gives a quite good adequacy of the model versus the experimental results (fig. 9).

The implementation of the constitutive law in the finite element code Abaqus has allowed one to compare simulations to more complex experimental configurations as three-point bend tests and Brazilian experiments. The global response of the compression diametrical test is given in fig. 11. One can see that too much nonlinearity appears in the model response. This point is in study, the three-point bend test giving providing first answers.

\section{Conclusion}

An experimental procedure has been carried out to characterize a complex material behaviour. A multibranch viscoelastic plastic and damageable model and the corresponding identification procedure have been developed. A genetic algorithm optimisation has one allowed to find accurately some of the viscous parameters. This model has been implemented in the finite elements software 
Abaqus using a user subroutine UMAT. The comparisons between simulations and experiments show a good agreement. Our future works are now devoted to the improvement of the damage rule and of the failure threshold. Lastly, the anisotropy observed during the experiments has to be introduced in the model. The pressure dependency of the viscosity is in study.

\section{Acknowledgement}

The authors address a special thanks to J.L. Brigolle for its contribution to this study, especially for the realization of the experiments.

\section{References}

[1] F.L. Addessio, J.N. Johnson, A constitutive model for the dynamic response of brittle materials, J. Appl. Phys., Vol. 67, 1990, pp 3275-3286.

[2] J.G. Bennett, K.S. Haberman, J.N. Johnson, B.W. Asay, B.F. Henson, A constitutive model for the non-shock ignition and mechanical response on high explosives, J. Mech. Phys. Solids, Vol. 46-12, 1998, pp. 2303-2322.

[3] R.M. Hackett, J.G. Bennett, An implicit finite element material model for energetic particulate composite materials, Int. J. Numer. Meth. Engng, Vol. 49, 2000, pp. 1191-1209.

[4] M. Gratton, V.D. Le, A. Frachon, M. Caliez, D. Picart, Mechanical behaviour of a viscoelastic plastic granular material: Experimental procedure and modelling, WSEAS trans. on comp., Is.1, Vol.5, Jan.2006, pp.149-156.

[5] M. Aubertin, L. Li, A porosity-dependant inelastic criterion for engineering materials, Int. Jour. of Plast., Vol. 20, 2004, pp. 2179-2208.

[6] J.H. Prevost, R. Popescu, Constitutive Relations for Soil Materials, Electronic Journal of Geotechnical Engineering, First Issue, 1996.

[7] O. Coussy, Mécanique des Milieux Poreux. Editions Technip, 1991.

[8] P.Y. Hicher, J-F. Shao, Elastoplasticité des sols et des roches - Modèles de comportement des sols et des roches - 1, Hermès Science Publications, 2002.

[9] F. Schleicher, Z. Angew, Math. Mech., 6:199, 1926.

[10] D.C. Drucker, A.M. Asce, R.E. Gibson, \& D.J. Henkel, Soil Mechanics and Work-Harding theories of plasticity, Transactions American Society of Civil Engineers, Vol. 122, 1957.

[11] X.D. Pan, J.A. Hudson, A simplified three dimensional Hoek-Brown yield criterion, Rock Mechanics and Power Plants, M. Romana (ed.), Rotterdam: Balkema, 1988, pp. 95-103.

[12] R. Raghava, R.M. Caddekk, G.S.Y. Yeh, The macroscopic yield behaviour of polymers, J. Material Science, Vol.8, 1973, pp. 225-232.

[13] D.E. Goldberg, Genetic algorithms in search, Optimization and Machine Learning, Addison Wesley Publishing Company, 1989.

[14] J. Mazars, Mechanical damage and fracture of concrete structure, Proc. I.C.F. 5, Cannes, France, 1981, pp. 1499-1506. 
[15] A. Dragon, D. Halm, Th. Désoyer, Anisotropic damage in quasi-brittle solids: modelling, computational issues and applications, Comput. Methods Appl. Mech. Engrg., 183, 2000, pp. 331-352.

[16] D. Halm, A. Dragon, An anisotropic model of damage and frictional sliding for brittle materials, Eur. J. Mech., A/Solids, Vol.17, no.3,1998, pp.439-460.

[17] J. Mazars, A description of micro and macroscale damage of concrete structures, Engineering Fracture Mechanics, Vol.25, no.5/6, 1986, pp.729-737.

[18] J. Mazars, F. Ragueneau, G. Pijaudier-Cabot, Continuum damage modelling for concrete structures in dynamic situations, Continuum Damage Mechanics of Materials and Structures, O. Allix and F. Hild (Editors), Elsevier Science Ltd., 2002.

[19] J. Lee, G.L. Fenves, Plastic-damage model for cyclic loading of concrete structures, Journal of Engineering Mechanics, vol.124, no.8, pp.892-900, 1998. 